Bei der gleichzeitigen Prüfung von verschiedenen Nervenextrakten mit dem Polarographen und am Froschherzen zeigte sich eine auffallende Parallelität zwischen polarographischer Aktivität und positiv inotroper Wirkung. Die Vermutung, es könnte mit den beiden verschiedenen Methoden ein und dieselbe Sub$\operatorname{stan} z$ erfaBt werden, wurde durch folgende Tatsachen crhärtet :

1. Nervenextrakte und Blutverdünnungen, die polarographisch die gleiche Aktivität besitzen, sind auch am Froschherzen gleich stark wirksam. Würden mit den zwei Methoden zwei verschiedene Substanzen er-

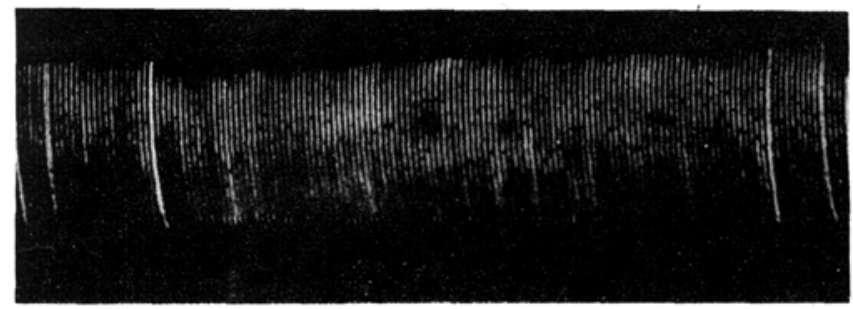

A $\quad$ B

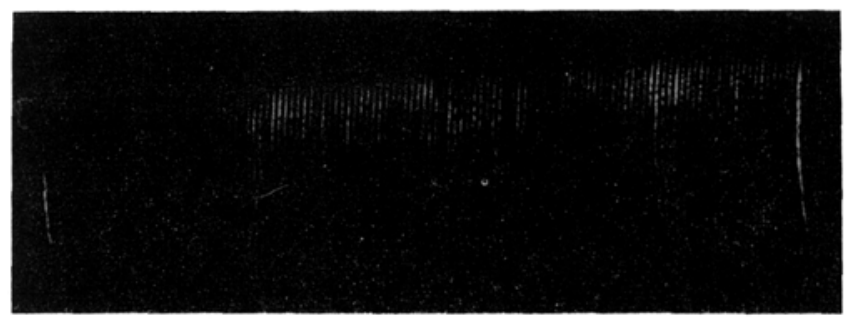

C D

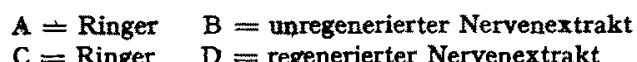

$\mathrm{C}=$ Ringer $\quad \mathrm{D}=$ regenerierter Nervenextrakt

faßt, so müßte man verlangen, daB die beiden Substanzen in Blut- und Nervenextrakten im gleichen Verhältnis vertreten wären.

2. Durch "alkalische" oder "saure" Regeneration (wie in der vorangehenden Mitteilung beschrieben), stieg in gleichem Maße wie die polarographische Aktivität auch die Wirkung am Froschherzen. Beispiel (siehe Kurve) : Ein Nervenextrakt ergab eine polarographische Aktivität von 1900 Einheiten vor und 2250 Einheiten nach der Regeneration. Das numerische Verhalltnis zwischen maximaler Hubhöhe mit Extrakt und der $H u b$ höhe mit Ringerlösung war 1,1 vor und 1,25 nach der Regeneration. Dieses Verhalten ist sicher so charakteristisch, daB es kaum mehreren Substanzen eigen sein kann. Durch Atropinisierung wird die Wirkung auf das Herz nicht beeinflußt.

Es erscheint somit höchst wahrscheinlich, daß die von LISSAK und die von v. MURALT entdeckte Substanz 1dentisch sind. Die praktische Bedeutung dieser Tatsache liegt darin, daß nunmehr die Identifizierung dieser biologisch interessanten Substanz mit einer einfachen, physikalisch-chemischen Methode angegangen werden kann.

S. WeIdManN und F. Wyss

Hallerianum, Bern, den 20. April 1945.

\section{Ergebnisse der Behandlung der Hyperthyreosen mit Thiouracil}

Amerikanische Forscher (Chesney, A. M., Clawson, Webster, Marine, Baumann u.a.m.) machten 1928 die Entdeckung, daß frischer Kohl, in großen Mengen an Kaninchen verfüttert, zu Kropfbildung führt. Dann veröffentlichte BLUM experimentelle Arbeiten, die bestätigen, daB im Kohl (verschiedene Brassicaarten) eine Noxe vorhanden ist, die beim Tier zu einer kropfigen Entartung der Schilddrüse führt. Die Kröpfe sind blutreich, jodarm und im Kaulquappenversuch inaktiv. Histologisch finden sich Vermehrung und Vergrößerung der Zellen, verunstaltete Follikel und Kolloidschwund. Fügt man dem Kohl kleine Jodmengen zu, so kann die Kropfbildung verhindert werden; gibt man das Jod erst nach Ausbildung des Kropfes durch die Kohlnoxe, so resultiert das typische Bild des Basedow.

KENNEDy fand 1942 als wirksamen Stoff im Rapssamen den Allylthioharnstoff. Von den verschiedenen chemischen Substanzen, die einer experimentellen Prüfung unterworfen wurden, zeigten sich vor allem Thioharnstoff und Thiouracil geeignet, die Bildung bzw. die Wirkung des Thyroxins zu verhindera. In der angloamerikanischen Literatur wird über etwa 200 Fälle berichtet, die vorzüglich mit Thiouracil behandelt wurden, und zwar mit täglichen Dosen his zu 1,0 g. Die Wirkung bei Hyperthyreose war zum Teil überraschend, doch schreckten Nebenerscheinungen, vor allem Fälle von Agranulozytose, zunächst von der weiteren Verwendung ab.

Seit einigen Monaten wurden in der Medizinischen Poliklinik der Universität Bern 12 Fälle von Hyperthyreose mit einem Thiouracilderivat behandelt, das uns von der Firma Wander, Bern, unter der Nr. "133» zur Verfügung gestellt wurde; das betreffende Präparat hat sich im biologischen Experiment als wirksamer und weniger toxisch erwiesen als das Thiouracil und kann deshalb $60 \%$ niedriger dosiert'werden. Eine Tablette enthält $0,04 \mathrm{~g}$ wirksame Substanz. Wir richten uns bei der Dosierung nach der Höhe des Grundumsatzes (2mal 2 bis 3 mal 3 Tabletten p. d., später Stabilisierungsdosen von $2-3$ mal 1 Tablette p. d.).

Bei unseren Patienten handelt es sich zum Teil um inveterierte Basedowfälle, wo die Kranken eine Operation abgelehnt hatten und schon während längerer Zeit in üblicher Weise mit Prominal, Luminal, Brom, Diät usw. behandelt worden waren. Bei etwa $3 / 4$ der Fälle konnten nach wenigen Wochen ganz auffällige, zum Teil überraschende Besserungen erzielt werden: Der Grundumsatz geht auf normale. Wèrte herunter, das Körpergewicht steigt, die Tachykardie nimmt ab, die Spontantöne verschwinden, Fingertremor, Schweißausbrüche und Muskelschwäche weichen. Die Konsistenz der Schilddrüse wird weicher. Es tritt in einigen Fällen eine Vergrößerung der Schilddrüse auf; die Gefäßgeräusche verschwinden; der Exophthalmus wird kaum beeinfluBt. Nebenerscheinungen: in zwei Fällen leichte Ekzeme; häufig Leukopenie mit relativer Lymphozytose, die nie schwerwiegender Natur und nur vorübergehend sind; einmal Erbrechen und Widerwillen gegen das $\mathrm{Me}$ dikament bei einer psychisch schwer alterierten Patientin. Uber die Wirkung des Präparates bei Herzinsuffizienz und bei Diabetikern fehlen uns noch schlüssige Resultate.

Die neue Behandlung der Hyperthyreose mit Thiouracilpräparaten ist in ihrem Wesen erstmalig und verblüffend.

W. HADORN

Medizinische Universitätspoliklinik, Bern, den 24. April 1945. 